\title{
Mesotelioma maligno de pleura com associação etiológica a asbesto: a propósito de três casos clínicos
}

\author{
E.M. de Capitani, K. Metze, C. Frazato J R., A.M.A. Altemani, L. Zambom, i.f.C. Toro, E. Bagatin \\ Hospital de Clínicas da Faculdade de Ciências Médicas da Universidade Estadual de Campinas (UnICAMP), Campinas, SP.
}

RESUMO - O mesotelioma maligno de pleura (MM) é tumor de ocorrência rara em nosso meio e tem-se mostrado, quando em elevada incidência, relacionado à exposição pregressa a asbesto ou amianto nas séries descritas em outros países.

Овјетіvo. A partir de casos clínicos atendidos no Hospital de Clínicas da UNICAMP, procurou-se evidenciar tal associação epidemiológica, visando alertar profissionais médicos quanto à possível elevação da incidência de tal tumor nesta década e, futuramente, por características próprias da história natural desse tipo de tumor.

Métodos. São descritos três casos de MM de pleura ocorridos na região de Campinas, SP, num período de dois anos, dos pontos de vista clínico, laboratoriais complementares e anatomopatológico, incluindo minuciosa investigação anamnésica ocupacional e ambiental.

Resultados. Todos os casos foram confirmados do ponto de vista anatomopatológico, incluindo mi croscopia eletrônica, como casos de MM. Os três casos mostraram relação epidemiológica, comprovada por meio de história ocupacional, com exposição a asbesto no passado. Um dos casos teve exposição ocupacional pregressa de curta duração

\section{NTRODUÇÃO}

Apesar de conhecido desde 1767, quando Lieutand relatou dois casos em cerca de 3.000 autópsias $^{1}$, a primeira descrição detal hada da patologia foi feita por Wagner, em 1870․ No entanto, somente neste século o mesotel i oma maligno (MM) foi caracterizado como enti dade nosol ógica específica, com critérios diagnósticos bem definidos ${ }^{2-4}$. Apesar dos inúmeros trabalhos publicados até o momento, o MM continua sendo um tema controverso na literatura científica. Coleman et al. consideram que o assunto permaneça em ativa discussão por três motivos: a) sua estreita associação etiológica com exposição ao asbesto, trazendo implicações legais e científicas de importância no campo da pesquisa de agentes e mecanismos de ação carcinogênicos; b) o fato de o MM representar um modelo de câncer em função de sua capaci dade multidirecional de diferenciação epitélio-mesen- (cerca de um ano); outro teve exposição doméstica a partir de asbesto trazido do ambiente de trabaIho por seu pai, durante sua infância, e o terceiro caso com contaminação ocupacional indireta.

Conclusão. A ocorrência de MM de pleura relacionada à exposição a asbesto, no passado, parece ser realidade em nosso meio, como mostram estes três casos clínicos. Destaca-se a necessidade da anamnese ocupacional e ambiental detalhadas na abordagem desses casos, alertando-se para maior atenção no diagnóstico dos tumores primários da pleura e do peritônio, nos próximos anos, em função de provável aparecimento de novos casos relacionados a exposição a asbesto pregressa, tendo em vista o intervalo de tempo entre o início da manipulação do asbesto em nosso país e o aparecimento destes primeiros casos coincidir com o tempo de latência médio esperado para a ocorrência de MM. Destacam-se, ainda, as necessidades de definição precisa de critérios diagnósticos, para esse ti po de tumor, e criação de registro centralizado de casos.

Unitermos: Mesotelioma maligno. Pleura. Asbesto. Etiologia. Diagnóstico.

quimal; e c) a dificuldade de definição diagnóstica em vida, a partir de fragmentos pequenos de biópsia, necessitando distinção entre hiperplasia inflamatória mesotelial e neoplasias metastáticas como o adenocarcinoma 5 .

Por definição, o mesotelioma é um tumor que provém das células mesoteliais multipotenciais da pleura ou peritôni o. Tem caráter rápi do, difuso e extenso de crescimento, envolvendo grande parte da superfície serosa afetada. Apresenta alto grau de malignidade, expresso por invasão local de partes moles, como parede torácica, parênquima pulmonar, pericárdio adjacente e linfonodos regi onais. Pode produzir metástases para os pulmões, fígado, pâncreas, rins, supra-renais e medula óssea6 ${ }^{6}$ com sobrevida média não ultrapassando doze meses, independentemente do tipo de tratamento realizado ${ }^{7}$. Classifica-se em três tipos histopatológi cos: epitelial, fibrossarcomatoso e misto ou bifásico ${ }^{6,7}$. 
O presente trabalho descreve três casos de mesotelioma maligno de pleura diagnosticados no Hospital de Clínicas da UNICAMP, no período de 1992-93, relacionados a exposições pregressas a asbesto, chamando a atenção para al guns aspectos epidemiológicos de importância para o entendimento da relação causal implícita e definição de políticas de prevenção mais agressivas no que se refere à exposição ao asbesto no Brasil, além de políticas de vigilância epidemiológica na área de Saúde Ocupacional.

\section{RELATO DOS CASOS}

Caso 1 - R.R., masculino, 32 anos, branco, apresentou-se pela primeira vez em serviço de atenção primária com queixa de dor torácica havia dois meses, em região ínfero-lateral de tórax direito, contínua, com piora à inspiração profunda e aos esforços. Negava febre, tosse ou dispnéia. Apresentava emagrecimento de $18 \mathrm{~kg}$ em seis meses, com astenia e inapetência. Tabagista de 36 anos/ maço. Etilista de 1/2 litro de conhaque por dia, nos últimos seis anos. Referia episódio de pneumonia (sic) há 16 anos. Pai morto por cardiopatia decorrente de problema pulmonar devido a inal ação de poeira em ambiente de trabalho (sic). Antecedentes ocupacionais: 1973-1975 - trabalhou como balconista de lanchonete; 1975-1978 - ajudante de fundição, trabalhando com moldes de areia em siderurgia, em Barra Mansa, RJ. A cada mês, ajudava na troca de revestimento dos fornos, destruindo a camada antiga e deteriorada de isolamento térmico de amianto (asbesto), preparando a nova massa de amianto a ser recolocada; 19781982 - operador de forno de el etrodo para produção de carbureto na fabricação de acetileno; 19821987 - garçom e recepcionista (quando começou a ingerir bebidas alcoól icas); 1987-1990 - operador de pá carregadeira em olaria; 1990-1992 - pintor de paredes e eletricista autônomo. O paciente referia que seu pai havia trabalhado nesse serviço de desmanche e reposição de amianto nos fornos da siderúrgica de Barra Mansa, de 1955 a 1980, e que sua irmã contou que o pai vinha do trabalho com roupa suja de poeira de amianto, pois não havia sistema de troca de roupa e banho no local de trabalho, àquela época.

Ao exame físico, apresentava-se descorado $+/ 4$, sem adenomegalias, afebril, levemente dispnéico. $\mathrm{PA}=130 / 90$ e $\mathrm{P}=96$. Bulhas rítmicas normofonéticas. Murmúrio vesicular diminuído globalmente em todo o hemitórax direito e abolido em base direita. Ausência de massas ou infiltrações de parede torácica. Fígado não palpável e baço não percutível e não palpável. Hipocôndrio direito doloroso à pal pação. Radiograma de tórax mostrava derrame pleural à direita. O líquido pleural mostrou-se sanguinolento, com 55.000 hemácias, 8.000 leucócitos com $15 \%$ de neutrófilos, $79 \%$ de linfócitos, $1 \%$ de eosinófilos e $0,5 \%$ de monócitos; proteínas de $5,4 \mathrm{~g} / \mathrm{dL}$; glicose $82 \mathrm{mg} / \mathrm{dL}$; coloração pel o Gram sem visualização de bactérias; cultura para BAAR negativa; citologia oncótica negativa. Hemograma: $\mathrm{Hb}=9,9 \mathrm{mg} \% \mathrm{Ht}=32$; reticulócitos = $0,4 \%$; plaquetas normais. Pesquisa de BAAR no escarro negativa em várias amostras. Teste de tuberculina $=0 \mathrm{~mm}$. Broncoscopia mostrava discreto processo inflamatório em mucosa de árvore brônquica direita. Citologia oncótica do lavado e escovado broncoalveolar mostrou-se negativa. Espirometria: $\mathrm{CVF}=2,0 \mathrm{~L}(50 \%) \mathrm{VEF}_{1}=1,87 \mathrm{~L}$ (56\%) $\mathrm{VEF}_{1} / \mathrm{CVF} \%=109 \%$. Gasometria: $\mathrm{pH}=7,437, \mathrm{pO}_{2}=$ $88,8, \mathrm{pCO}_{2}=35,4, \mathrm{HCO}_{3}=24,0, \mathrm{~EB}=+0,8, \mathrm{SatO}_{2}=$ 97 ,2. $\mathrm{ECG}=$ sinusal. Biópsia de pleura por toracotomia em 23/3/92 mostrou neoplasia maligna do tipo epitelial com arranjo glandular. Coloração pelo PAS diastase foi negativa; CEA negativo e EMA positivo, apontando para o diagnóstico de mesotelioma maligno de pleura. Diagnóstico definitivo realizado com fragmentos provenientes da peça cirúrgica por meio de microscopia eletrônica que mostrou presença de microvilosidades características de células neoplásicas mesoteliais. Diagnóstico definitivo: mesotelioma maligno de pleura do tipo epitelial.

Caso 2 - J.N.M., masculino, 54 anos, branco, procurou o serviço em maio de 1992 com queixa de dispnéia, cansaço e emagrecimento de $6 \mathrm{~kg}$ em cerca de oito meses. Referia dor torácica ventilatório-dependente à direita e tosse com expectoração amarelada. De antecedentes pessoais, era proveniente de zona endêmica de doença de Chagas; duas cirurgias para úlcera péptica havia dez anos; hipertensão arterial moderada em uso de nifedipina. Tabagista de 20 anos/maço. Antecedentes ocupacionais: trabalhou durante toda sua vida como escriturário em setores administrativos, exceto entre 1967 e 1968, quando se empregou na produção de fibrocimento em Leme, SP, por período de 12 meses.

Ao exame físico, apresentava-se em bom estado geral e eupnéico. PA $=140 / 90$ e $P=72$, sem adenopatias; ausência de massas em tórax; murmúrio vesicular abolido em base direita até campo médio; fígado não palpável; baço não percutível. Radiograma de tórax mostrava opacidade homogênea, sem broncograma aéreo, abrangendo todo o hemitórax direito compatível com derrame pleu- 


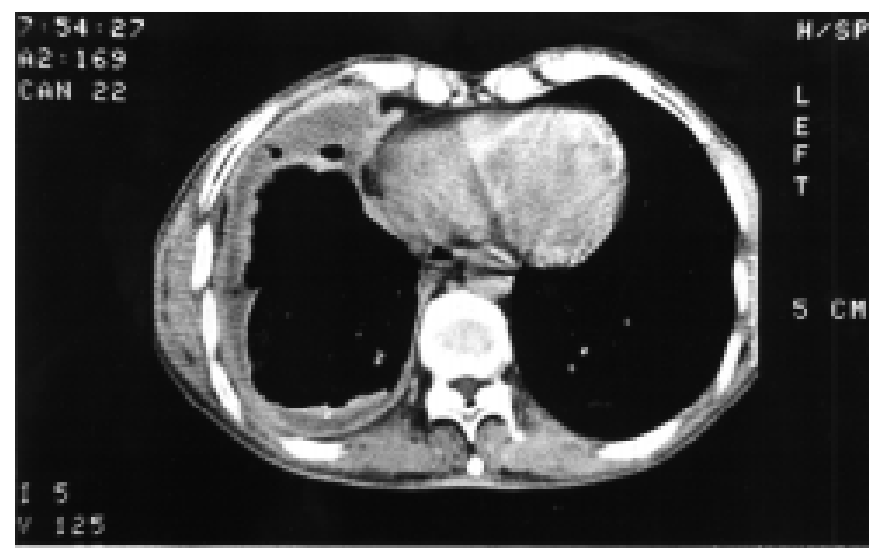

Fig. 1 - Tomografia computadorizada de tórax (caso 2) mostrando espessamento pleural intenso com redução volumétrica de pulmão $\mathrm{D}$ e acometimento de parte do pericárdio.

ral, com discreto desvio de mediastino para a esquerda. Após esvaziamento do derrame, observou-se espessamento pleural difuso, mais intenso em base, sem imagens alteradas em parênquima à esquerda. Tomografia computadorizada de tórax evidenciou redução volumétrica de todo o pulmão direito às custas de intenso espessamento da pleura, mediastino livre e ausência de lesões parenquimatosas (fig. 1). Líquido pleural: $\mathrm{pH}=7,7$, densidade $=1.030$; proteínas $=3,6 \mathrm{~g} / \mathrm{dL} ;$ glicose $=$ $11 \mathrm{mg} \%$; hemácias = 310; leucócitos $=107$, sendo $93 \%$ linfócitos, 3\% neutrófilos, 1\% eosinófilos e $1 \%$ monócitos; células mesoteliais com intensa vacuolização; citologia oncótica classe II; col oração pelo Gram não visualizou bactérias. Broncoscopia sem alterações. $\mathrm{Hb}=13,4 \mathrm{mg} \% ; \mathrm{Ht}=38,5$; CEA no sangue $=2,8$; citologia oncótica de escarro = classe $1 \mathrm{I}$; cultura para BAAR no escarro =negativa. Biópsia de pleura de 29/5/92 evidenciou quadro histopatológico de mesotelioma maligno de pleura do tipo epitelial. Em função de recorrência do derrame pleural, optou-se por pleurodese, realizada em junho de 1992, e quimioterapia com cysplatinum $120 \mathrm{mg} / \mathrm{m}^{2}$ e etoposida $300 \mathrm{mg} / \mathrm{m}^{2}$, seguida de radioterapia.

Caso 3 - M.S.A., masculino, 54 anos, branco, procurou o serviço de Cirurgia Torácica com dor em hemitórax direito nos três meses anteriores. Contava que há cerca de dois anos sentia dor em pontada na base anterior de hemitórax direito, a princípio relacionada a esforços, com piora progressiva até três meses atrás, quando apresentou febre e pneumonia (sic) com derrame. Após tratamento, a febre cessou, mas manteve-se o derrame, quando compareceu ao serviço para diagnóstico. Referia astenia e cansaço, com emagrecimento de $20 \mathrm{~kg}$ em três meses. Tabagista de 72 anos/maço. Pneumonia há oito

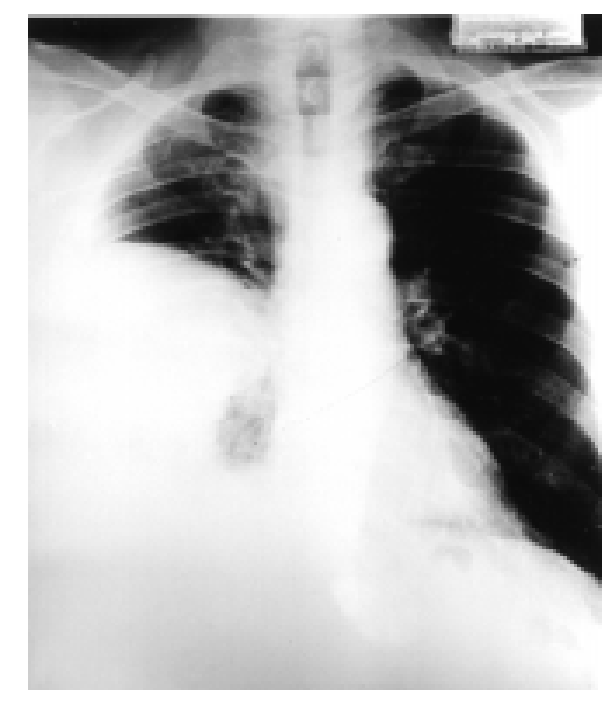

Fig. 2

Radiograma simples de tórax (caso 3) mostrando opacidade homogênea em hemitórax D acometendo os 2/ 3 inferiores.

anos; gastrectomia há 35 anos. Antecedentes ocupacionais: trabalhou inicialmente na lavoura e em seguida entrou no ramo de comércio, quando desenvolveu atividades de compra, venda e distribuição de pneus, até aposentar-se. Nesse período de comércio, houve um intervalo, nos anos de 1969 a 1971, quando trabalhou em pequena empresa de vulcanização de pneus, na cidade de Leme, SP. Essa empresa localizava-se, na época, a cerca de 300 metros de importante fábrica de fibrocimento, na qual o paciente passava algumas horas, todos os dias, por conhecer seus proprietários.

Ao exame físico, encontrava-se em bom estado geral, sem adenopatias. PA $=125 / 80$ e $P=80$. Murmúrio vesicular diminuído em base direita e abolido em região axilar e base anterior direita, com estertores subcrepitantes em campo médio direito. Radiograma de tórax mostrava opacidade homogênea abrangendo terços médio e inferior de hemitórax direito, com indefinição de diafragma nesse lado; massa mostrava ausência de contigüidade com mediastino; pulmão e pleuras à esquerda sem alterações; traquéia centrada (fig. 2). Tomografia computadorizada de tórax mostrava extensa massa acometendo hemitórax dir eito, aparentemente não-parenquimatosa, com extensão para parede torácica; áreas de atelectasia e derrame pleural; ausência de planos de clivagem com estruturas vasculares, e mediastino com gânglios acometidos (fig. 3).

Ultra-sonografia mostrava massa heterogênea, predominantemente sólida, com áreas císticas em seu interior, estendendo-se da região posterior da cavidade torácica até o diafragma ântero-lateralmente à direita, com deslocamento do fígado para baixo sem invadi-lo, dando impressão ecográfica de um sarcoma de parede. Gasometria: $\mathrm{pH}=7,476$, 


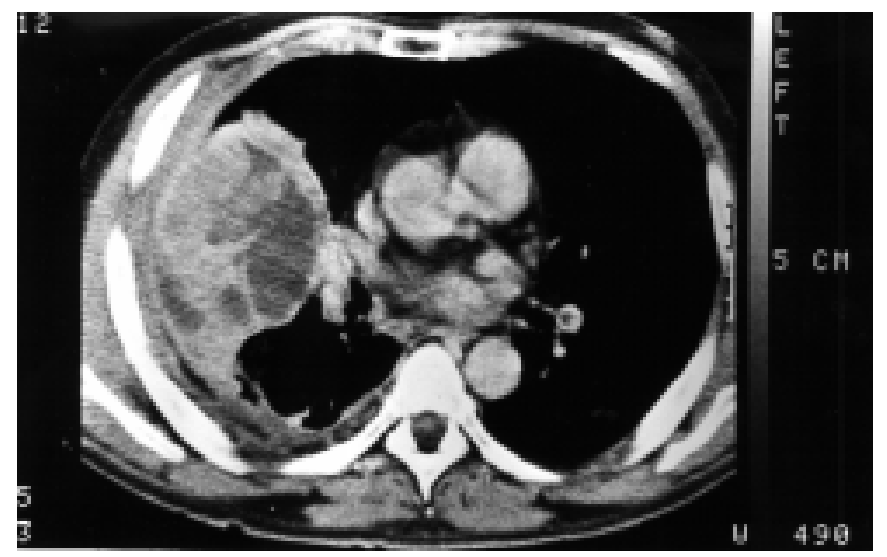

Fig. 3 - Tomografia computadorizada de tórax (caso 3) mostrando opacidade de ti po massa em hemi tórax $D$, em contigüidade com estruturas mediastinais, presença de gângli os comprometi dos no mediastino e acometi mento de parede torácica.

$\mathrm{pO}_{2}=72,3, \mathrm{pCO}_{2}=32,3, \mathrm{HCO}_{3}=24,0, \mathrm{~EB}=+1,6$, Sat $\mathrm{O}_{2}=95,6 ; \mathrm{Hb}=11,3 \mathrm{mg} ; \mathrm{Ht}=36$. Biópsia por toracotomia em maio de 1993, real izada em serviço externo, mostrou apenas tratar-se de neoplasia maligna fusocelular, sugestiva de fibro-histiocitoma (sic), porém sem definição diagnóstica definitiva. Realizada toracotomia para ressecção tumoral no dia 4/8/93. Anatomopatológico da peça cirúrgica mostrou tratar-se de mesotelioma maligno de pleura do tipo fibrossarcomatoso com PASdiastase negativa e CEA negativo. Análise pela microscopia eletrônica evidenciou microvilosidades características (fig. 4).

\section{DISCUSSÃO E CONCLUSÃO}

O mesotelioma maligno é tumor de ocorrência rara, com incidência esperada em populações de referência de cerca de 1 a 2 casos por milhão de habitantes por ano ${ }^{8}$. Incidências mais elevadas têm sido observadas em diversas áreas do mundo relacionadas a exposição ocupacional, doméstica e ambiental a asbesto. Por exemplo, na África do Sul, onde existe extensa mineração de crocidolita, a incidência de mesotel ioma maligno é de 7,2 casos por milhão por ano ${ }^{9}$. Da mesma forma, em área de mineração na Austrália, a ocorrência observada é de 15,8 casos por milhão ${ }^{10}$. Nos EUA, onde a manipulação de asbestos foi muito intensa no período em torno à Segunda Guerra Mundial, a incidência esperada na população adulta masculina está estimada entre 7 e 13 por milhão por ano ${ }^{11}$. Na Inglaterra, dados de 1968 a 1983 mostram que, quando as taxas são corrigidas por idade, não se observa aumento na incidência em mulheres com menos de 55 anos e em homens com menos de $45^{12}$, apesar da

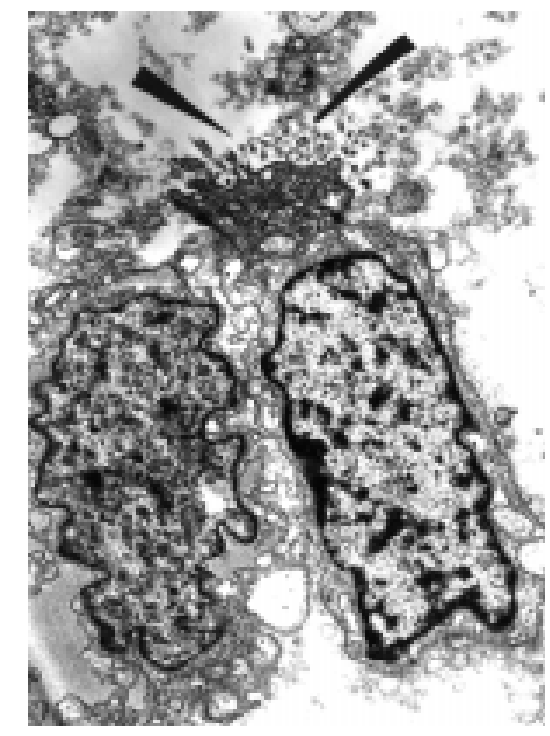

Fig. 4 -

Microscopia el etrônica mostrando detalhe de microvilosidades típicas que diferenciam o mesotel ioma maligno de outros tumores (setas). Célula mesenquimatosa. Aumento de 8.998x.

ocorrência geral de MM nesse período ter sido de 20/milhão entre os homens e 3/milhão entre as mulheres. Esse fato mostra que a ocorrência foi maior na faixa de idade produtiva, assinalando a existência de fator etiológico específico, no caso o asbesto $^{12}$. É consenso na literatura que ocorrências de Mм acima do esperado devem estar relacionadas com algum tipo de exposição pregressa a asbesto ${ }^{7,13,15}$.

O primeiro estudo de casos de $\mathrm{MM}$ relacionando excesso de ocorrência, com exposição a asbesto no passado, foi publicado por Wagner et al., em 19604. Descreveram 33 casos ocorridos num período de quatro anos, 32 deles provenientes de região mineradora de crocidolita na Província do Cabo, na África do Sul. As exposições ao asbesto foram caracterizadas como de tipo ocupacional (trabalho nas minas), e ambiental (nascimento e/ou moradia próxima às áreas de mineração). Apenas um caso não tinha definição de exposição de qual quer tipo ${ }^{4}$. Esse trabalho trouxe comoção nos meios científico e leigo de opinião pública, na época, em função dessa associação etiológica com doses aparentemente baixas de asbesto ${ }^{14}$, e confirmou indícios dessa associação já indicadas por Mallory, em 1947, e Wyers, em 194915,16. Cinco anos depois, Newhouse e Thompson, mediante estudo de casocontrole, estabeleceram três categorias de possível exposição pregressa relacionadas à ocorrência excessiva de mesoteliomas malignos: ocupacional, doméstica (exposição a asbesto trazido para a residência por pessoa ocupacionalmente exposta) e ambiental ${ }^{17}$. Desde então, essas três categorias vêm sendo confirmadas, do ponto de vista epidemiológico, como exposições a asbesto possíveis de explicar elevadas incidências regionais de $M M^{13,18}$. 
No Brasil, a manipulação industrial de asbesto remonta ao inicio do século, mas em pequena escala e com boa propor ção de asbesto importado ${ }^{19}$. A exploração mineral do asbesto, principalmente do tipo crisotila, iniciou-se, também em pequena escala, em 1920. A primeira mina de médio porte no Brasil iniciou atividades em 1940, no nordeste da Bahia, produzindo 3.000 toneladas $/ a_{n o}{ }^{20}$. Um pouco de antofilita foi explorado em Alagoas, na década de 60, mas a exploração, em grande escala e economi camente viável, começou em 1967 com a jazida de Canabrava, em Minaçu (GO), onde se encontram, ainda, extensas reservas de crisotila praticamente sem contaminação geológica com outras variedades de asbesto ${ }^{19,21}$. Até o inicio da expl oração de Canabrava, e mesmo nas décadas de 70 e 80, houve importação de quanti dades consideráveis de crocidolita e amosita para consumo das indústrias de fibrocimento, pastilhas de freios e indústrias de cloro-soda ${ }^{19}$.

A zeolita, fibra natural do tipo erionita, também está relaci onada a ocor rência de MM em populações expostas, tendo em vista o el evado número de casos incidentes na região da Anatolia, na Turquia, onde o solo é rico nesse tipo de fibra, e os moradores utilizam-se de matéria-prima local para construção de suas residências ${ }^{22}$.

Quanto às evidências experimentais e estudos de mecanismos de ação carcinogênica, os resultados da maioria dos trabalhos convergem para a confirmação da hipótese de Mearl Stanton, que relaciona a ocorrência de MM em animais de experimentação ao tamanho e durabilidade da fibra, incluindo na listagem de agentes causais, al ém do asbesto e da erionita, fibras sintéticas com características de tamanho e de depuração pulmonar comparáveis a certos tipos de asbesto ${ }^{23}$. Segundo essa hipótese, fibras del gadas menores de $0,25 \mu \mathrm{m}$ em diâmetro e maiores de $8 \mu \mathrm{m}$ de comprimento têm condições de chegar a nível alveolar e pleural, ali permanecendo por longo período de tempo, suficiente para desencadear o processo de carcinogênese. Dentro dessa hi pótese, as fibras de asbesto seguiriam uma hierarquia de potencial carcinogênico a partir da crocidolita, com maior potencial, seguida da amosita, crisotila e antofilita ${ }^{6,18}$. Ocorre que a exposição a mais de um tipo de fibra é freqüente e parece que a incidência de $M M$ em expostos a crisotila estaria relacionada a contaminação desta com fibras como a amosita, tremolita e antofilita ${ }^{24}$. A zeolita também preenche os critérios de Stanton, corroborando os achados epidemiológicos na Turquia. Outras possíveis causas de MM, de menor importância em termos de número absoluto de casos, relacionam-se com exposição a fibras artificiais não-asbesto, cicatrizes, processos inflamatórios crônicos e radiação ionizante ${ }^{25}$. Em extensa revisão sobre o tema, Peterson et al. citam diversos casos publicados de pacientes que se submeteram a radioterapia e apresentaram мM anos depois ${ }^{25}$. O tabagismo parece não estar associado a aumento de incidência de $M M$, e também não funci onaria como cofator em sua indução ${ }^{26,27}$.

Os três casos apresentados preenchem os critérios estabelecidos para diagnóstico definitivo de MM, ou seja, provas histoquímicas compatíveis e morfologia típica à microscopia eletrônica, ${ }^{5,6,28-30}$, que o diferenciam, por exemplo, do adenocarcinoma metastático. Além do adenocarcinoma, o MM pode ser confundido com fibrossarcoma, fibrohistiocitoma, timoma e carcinoma bronquíolo alveolar ${ }^{5,28}$. Em função dos aspectos legais envolvidos a partir do diagnóstico definitivo e das dificuldades diagnósticas diante de outras neoplasias e proliferação mesotelial reacional inflamatória, na Europa e na América do N orte existem comitês de especialistas credenciados para confirmar os casos de MM diagnosticados nos diversos serviços ${ }^{31,32}$.

Do ponto de vista epidemiológico, os três casos preenchem a maioria dos aspectos marcantes característicos da história natural do MM. O tempo de latência foi de 24 anos para os casos 2 e 3, e ao redor de 30 anos para o caso 1 , desde que se considere a contaminação ocorrida na infância a partir do asbesto trazido para casa pelo pai, na década de 50. Análise de dados disponíveis envolvendo informações sobre dose de asbesto inalada tende a mostrar que o tempo de latência (definido como o tempo decorrente entre a primeira exposição e a ocorrência do efeito) médio para o mM é, aproximadamente, o mesmo para trabalhadores expostos por curto tempo ou por longos perío$\operatorname{dos}^{33-35}$. Nos casos de exposições muito intensas, parece haver pouco ou nenhum aumento do risco relativo de ocorrência de MM depois de dois anos de exposição, indicando que, para alguns trabaIhadores, o desenvolvimento do MM está, provavelmente, ligado à inalação de fibras de asbestos ao início da exposição, como nos casos 2 e $3^{36,37}$. Trabalho de coorte prospectivo, realizado por Selikoff et al. em isoladores térmicos norte-americanos, mostra tempo de latência entre 25 e 40 anos em 175 casos de MM $^{38}$.

Quanto ao tipo de contaminação ocorrida, podemos classificar os três casos, respectivamente, em domiciliar (caso 1), ocupacional (caso 2) e

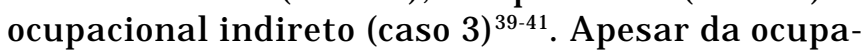
ção relatada no caso 3 não se relacionar, etiologicamente, com MM, a freqüência quase que diária a um ambiente de trabalho contaminado por asbes- 
to, durante quase três anos, por motivos extraocupacionais, não anula as características ocupacionais indi retas de exposição a que se submeteu o paciente. No que se refere ao número de casos incidentes esperados para determinada população, os casos 2 e 3 são ambos provenientes de uma cidade da região de Campinas, com cerca de 60.000 habitantes, na qual a incidência esperada seria de nenhum caso $^{8}$. Essa incidência de dois casos em 2 anos numa dada região (no caso a mesma cidade) caracteriza situação de ocorrência em cluster, chamando a atenção para possíveis novos diagnósticos, a partir da mesma área geográfica, desde que ambos os casos foram caracterizados etiologicamente como secundários a exposição a asbesto em ambiente industrial, em atividade largamente difundida nessa locali dade desde a década de $60^{42}$. Cal cula-se que cerca de 1.000 a 1.500 trabal hadores estariam expostos a asbesto nas indústrias da região ${ }^{43}$. No Brasil, as estimativas variam de 20.500 a 28.000 trabal hadores expostos em algum tipo de atividade ligada à manipulação de asbesto ${ }^{19,44}$.

Quanto à literatura nacional, dos três casos publicados por Franco et al. em 1985, apenas um deles relatava exposição a asbesto, por 20 meses, a partir de $1977^{45}$. Os outros dois casos careciam de história ocupacional detalhada e de provas histoquímicas comprobatórias. Da mesma forma, Azevedo et al., em 1985, publicaram relato de quatro casos de $M M$, todos com sumária história profissional, aparentemente sem exposi ção a asbesto, apesar de terem encontrado asbestose (sic) em gânglio hilar, no histopatológico de necrópsia de um dos $\operatorname{casos}^{46}$. Em 1989, Choma et al. relataram caso de rapaz de 22 anos com MM de tipo misto, sem história de exposição a asbesto no trabalho. Do relato não consta a história ocupacional dos pais, o que teria importância, tendo em vista a pouca idade do paciente ${ }^{47}$.

Com relação ao tipo de asbesto envolvido na ocorrência dos casos relatados, o asbesto minerado em grande escala no Brasil, e consumido pela maior parte das indústrias de fibrocimento do país, atualmente, é do tipo crisotila com al to grau de pureza ${ }^{19}$, proveniente da mina de Canabrava, GO. Segundo a escala de Stanton (1988) ${ }^{23}$, confirmada por diversos autores ${ }^{13}$, a crisotila estaria em terceiro lugar, em potencial de causar MM, e mesmo assim, provavelmente, em função de contaminação com amosita ou tremolita ${ }^{13,14,24}$. No entanto, no Brasil das décadas de 50 e 60 , antes do pleno funcionamento da mina de Canabrava, grande proporção do asbesto para mani pulação industrial era importada, incluindo crocidolita e amosita19,21. Até 1960 , cerca de $85 \%$ do consumo de asbesto no
Brasil, que era de cerca de 20.000t/ano, era proveniente de asbesto importado ${ }^{20}$. São comuns os depoi mentos de trabal hadores, que manipularam asbesto nessas épocas, referindo-se ao asbesto de cor azul (crocidolita) proveniente de países estrangeiros. Assim, não é infundada a hipótese de ter havido contami nação com crocidolita por parte dos três pacientes com MM aqui relatados.

O caso 1 , referente a provável contaminação doméstica por fibras de asbesto, trazidas do ambiente de trabalho pelo pai do paciente, durante prolongado período de tempo, tem diversos correlatos na literatura internacional; este tipo de contaminação já havia si do estabel eci do epi demi ol ogicamente, em 1965, por Newhouse \& Thompson, e continua a ser comprovada por vários relatos publicados desde então ${ }^{39,40,48}$.

Do ponto de vista terapêutico, a cirurgia de escol ha para casos de MM é a pleuropneumectomia com retirada do hemidiafragma e pericárdio ${ }^{49}$. Esse procedimento registra mortalidade cirúrgica em torno de $10 \%$, e só foi possível ser realizado no caso 1 , pois o caso 2 não apresentava condições clínicas adequadas e, no caso 3, optou-se por bilobectomia, apenas em função do diagnóstico histopatológico inicial de fibro-histiocitoma.

Os dados disponíveis na literatura, até o momento, são insuficientes ou inadequados para permitir avaliação da eficácia da quimi oterapia nesses tipos de caso ${ }^{6}$. Diversas drogas têm sido usadas com variados graus de resposta terapêutica, tais como cicl ofosfamida, metrotrexato, 5-fluorouracil, cisplatina e doxorubicina ${ }^{49-53}$. A associação dos tratamentos cirúrgico, quimioterápico e radioterápico não parece trazer vantagens na sobrevida média, quando se compara cada tratamento independentemente. O MM parece insensível à radioterapia ${ }^{54}$.

Com referência ao prolongado tempo de latência desse tipo de tumor, espera-se aumento substancial de ocorrência dessa enfermidade para os próximos anos, em função da história da manipulação do asbesto no país ${ }^{19,20}$, especialmente nas regiões da Grande São Paulo e Campinas, sedes de empresas de fibrocimento, além das cidades portuárias, onde se localizam grandes estaleiros e que utilizam isolamento térmi co à base de asbesto. A redução dos níveis de exposição nos ambientes de trabalho a partir de medidas de controle coletivo, como exaustão e manipulação industrial adequadas, bem como de cronograma efeti vo de substituição do asbesto por materiais menos insalubres, é tarefa inadiável das empresas e dos setores de definição de políticas de saúde pública e fiscalização, visando prevenir ocorrências futuras de MM e outras doenças relacionadas à exposição ao asbes- 
to. Existe, além disso, a necessidade de maior precisão nos diagnósticos dos tumores primários de pleura e peritônio, bem como no detalhamento da história ocupacional dos acometidos, no intuito de esclarecer provável relação causal com o asbesto nos novos casos que venham a ocorrer. Nesse sentido, seria oportuno instituir um registro especial e centralizado de todos os casos de MM ocorridos no país, com definição precisa de critérios diagnósticos e de investigação etiológica através de história ocupacional do paciente, de parentes, e história ambiental direcionada, e mediante análise de resíduos inorgânicos (fibras) em tecidos provenientes de biópsias e autópsias ${ }^{24}$.

O co-autor K.Metze é pesquisador do CN Pq.

\section{SUMMARY}

\section{Diffuse malign mesothelioma of pleura etiolo- gically related to asbestos exposure: discussion of three clinical cases}

Diffuse Malign Mesotheliomas (DMM) has a low background prevalence. High incidences of this tumor have been related to asbestos exposure in the past.

Purpose. To describe and discuss three clinical cases treated in our hospital, in which precise histopathologic diagnosis was made, and detailed occupational and environmental histories were taken, trying to identify in their past some kind of asbestos exposure.

METHODS. Three cases of DMM are described. Diagnosis was confirmed by histochemical analysis and el ectronmicroscopy. Detail ed occupational and environmental histories were taken from subjects and their families, searching for past contact with asbestos.

RESULTS. The cases were diagnosed in a short period of time (two years), in a region of the country where many asbestos cement plants are located since the mid sixties. Skillful histological procedures were used. From these cases we found out that one had a twelve months period of exposure, 24 years before, in one of those plants. Another patient had an exposure for three years, as a bystander, in the same plant (also 24 years before) and a third patient was contaminated by asbestos brought home by his father in the 1950s (latency period of 30 years). All cases were histochemically studied and diagnosis confirmed by the presence of microvilli at el ectronmicroscopic examination.

Conclusions. These three cases seem to confirm the existence of the epidemiologic association with asbestos exposure in our country. Definition of diagnosis criteria, centralization of cases registry and the necessity of more attention to this kind of asbestos related disease are discussed and stressed, as many new cases like those described are thought to occur in the near future, as the latency period of the disease seems to match with that of industrial asbestos manipulation in Brazil. [Rev Ass Med Brasil 1997; 43(3): 265-72.]

KEY WORDS: Diffuse malign mesothelioma. Pleura. Asbestos. Etiology. Diagnosis.

\section{REFERÊNCIAS BIBLIOGRÁFICAS}

1. Robertson HE. Endotelioma of the pleura.J Cancer Res 1924; 8:317-75.

2. Dubray ES, Rosson FB. Primary mesothelioma of the pleura. A clinical and pathological contribution to pleural malignancy, with a report of case. Arch Intern Med 1920; 26: 715.

3. Klemperer $\mathrm{P}$, Rabin CB. Primary neoplasma of the pleura. Arch Pathol 1931; 11: 385-412.

4. Wagner J C, Sleggs CA, Marchand P. Diffuse pleural mesothelioma and asbestos exposure in the north-western Cape Province. Br J Ind Med 1060; 17: 260-71.

5. Coleman M, Henderson DW, Mukherjee TM. The UItrastructural pathology of malignant pleural mesothelioma. Pathol Ann 1989; 24: 303-53.

6. Enzinger FM, Weiss SW. Soft tissuetumors, $3^{\text {rd }}$ ed. St Louis, Mosby, 1995; 787-819.

7. Lilis R. Mesothelioma. In Merchant J A (ed): Occupational respiratory diseases, Washington, USDHHS-CDC-NIOSH, 1986; 671-88.

8. Pelnar PV. Asbestos (mesothelioma and Iung cancer). In Parmeggiani $L$ (ed): Encycl opaedia of occupational health and safety, $3^{\text {rd }}$ ed. Geneva, I.L.O, 1983; 191-7.

9. Solomons K. Malignant mesothelioma: clinical and epidemiological features. A report of 80 cases. S Afr Med J 1984; 66: 407-12.

10. Leigh J , Corvalan CF, Grimwood A et al. The incidence of malignant mesothelioma in Australia 1982-1988. Am J Ind Med 1991; 20: 643-55.

11. Spirtas R, Beebe GW, Connely RR et al. Recent trends in mesothelioma incidence in the United States. Am J Ind Med 1986; 9: 397.

12. J ones R, Smith DM, Thomas G. Mesothel ioma in Great Britain in 1968-83. Scand J Work Environ Health 1988; 14: 145-52.

13. Wagner J C. The discovery of the association between blue asbestos and mesothelioma and the aftermath. $\mathrm{Br} J$ Ind Med 1991; 48: 399-403.

14. Murray R. Asbestos: a chronology of its origins and health effects. Br J Ind Med 1990; 47: 361-5.

15. Mallory TB, Castleman B, Parris EE. Case records of Massachusets General Hospital/ Case 33111. N Engl J Med 1947; 236: 407-12.

16. Wyers H. Asbestosis. Postgrad Med J 1949; 25: 631-38.

17. Newhouse $M L$, Thompson $H$. Mesothelioma of pleura and peritonium foll owing exposureto asbestos in the London area. $\mathrm{Br} J$ Ind Med 1965; 22: 261-9.

18. Rom WN. Asbestos-related diseases. In Rom WN (ed): Environmental and Occupational Medicine, $2^{\text {nd }}$ ed. Boston-Toronto-London, Little, Brown \& Co, 1992; 269-91.

19. N ogueira DP. O asbesto e sua utilização no Brasil. Rev Bras S Ocup 1988; 16: 7-9. 
20. Berman DM. Asbestos and health in the third world: the case of Brazil. Int J Health Serv 1986; 16: 253-63.

21. Nunes J SM. As atuais condições de utilização do asbesto no Brasil. Rev Bras S Ocup 1988; 16: 34-7.

22. Baris YI, Sahin A, Ozemi M et al. An outbreak of pleural mesothelioma and chronic fibrosing pleurizy in the village of Karain/U rgup in Anatolia. Thorax 1978; 33: 181-92.

23. Stanton MF, Layard M, Tegeris A et al . Relation of particle dimension to carcinogenicity in amphibol easbestos and other fibrous minerals. J Natl Cancer Inst 1981; 67: 965-75.

24. Churg A. Fiber counting and analysis in the diagnostis of asbestos-related disease. Hum Pathol 1982; 13: 381-92.

25. Peterson J T, Greenber SD, Buffler PA. Non-asbestos-related malignant mesothelioma: a review. Cancer 1984; 54: 951-60.

26. McDonald AD. E pidemiology of primary malignant mesothelial tumors in Canada. Cancer 1970; 26: 914-9.

27. McDonald AD, MCDonald J C. Malignant mesothelioma in North America. Cancer 1980; 46: 1.650-6.

28. Kannerstein M, Churg J, McCaughey WTE. Asbestos and mesothelioma: a review. Pathol Ann 1978; 13: 81-129.

29. Battifora $\mathrm{H}$, Kopinsk MI. Distinction of mesothelioma from adenocarcinoma. An immunohistochemical approach. Cancer 1985; 55: 1.679-85.

30. Pfaltz M, Odermatt B, Christen B, Rutter J R. Immunohistochemistry in the diagnosis of malignant mesothelioma. Virchows Arch 1987; 411: 387-93.

31. McDonald AD, Magnes D, Eyssen G. Primary malignant mesothelial tumors in Canada 1960-68. Cancer 1985; 55: 1.679-785.

32. Greenberg M, Loyd-Davies TA. Mesothelioma register 196768. Br J Ind Med 1974; 31: 91-104.

33. Browne K, Smither WJ. Asbestos-related mesothelioma: factors discriminating between pleural and peritoneal sites. Br J Ind Med 1983; 40: 145-52.

34. Mowe G, Gylseth B, Hartveit F, Skang V. Occupational asbestos exposure, lung fibre concentrations and latency time in malignant mesothelioma. Scand J Work Environ Health 1984; 10: 293-8.

35. Lanphear BP, B uncher CR. Latent period for malignant mesothelioma of occupational origin.J Occup Med 1992; 34: 718-21.

36. Seidmen H, Selikoff IJ , Hammond EC. Short-term asbestos work exposure and long term observation. Ann NY Acad Sci 1979; 330: 61-89.

37. Browne K. Asbestos-related mesothelioma: epidemiological evidence for asbestos as a promoter. Arch Environ Health 1983; 38: 261-6.

38. Selikoff IJ , Hammond EC, Deidman H. Mortality experience of insulation workers in the United States and Canada. Ann NY Acad Sci 1979; 330-91.

39. Grandjean $P, B$ ach E . Indirect exposures: the significance of bystanders at work and at home. Am J Ind Med 1986; 47 : 819-24.

40. Knishkowy B, Baker EL. Transmission of occupational disease to family contacts. Am J Ind Med 1986; 9: 543-50.

41. Kilburn KH, Lilis R, Anderson $\mathrm{HA}$ et al. Asbestos disease in family contacts of shipyard workers. Am J Publ Health 1985; 75: 615-7.

42. Riani Costa, J L. Asbestose: um exemplo de abordagem alternativa das doenças profissionais no Brasil. Rev Bras S Ocup 1984; 48: 7-19.

43. Lima FGA, AragãoJ RNM, Bonciani M et al. As condições de utilização doasbestonas indústrias defibrocimento do Estado de São Paulo. Rev Bras S Ocup 1988; 16: 41-50.

44. Riani Costa J L, Ferreira J r YM. As doenças relacionadas ao asbesto (amianto). Rev Bras S Ocup 1984; 47: 21-30.

45. Franco CAB, Silva RN, Made K, Sayeg F, Bethlem NM. Mesoteliomas pleurais. Apresentação de três casos e revisão da terapêutica. J Pneumol 1985; 11: 141-8.

46. Azevedo CM, Matushita J PK, Toscana E, Carvalho WR. Mesotelioma maligno de pleura. Radiol Bras 1985; 18 : 127-33.

47. Choma L, Gapski D, Pelanda LG, I osshi SO, Moreira Fo O. Mesotelioma maligno de pleura. J Pneumol 1989; 15: 171-4.

48. Cazzadori A, Malesani F, Romeo L. Malignant pleural mesothelioma caused by non-occupational childhood exposure to asbestos. Br J Ind Med 1992; 49: 559.

49. Antman KH. Malignant mesothelioma. N Engl J Med 1980; 4: $200-2$.

50. Antman $\mathrm{KH}$, Blum RH, Greenberg J S et al. Multimodality therapy for malignant mesothelioma based on a study of natural history. Am J Med 1980; 68: 356-60.

51. Adans VI, Unni KK, Muhn J R et al. Diffuse mesothelioma of thepleura: diagnosis and survival in 92 cases. Cancer 1986; 58 : 1.540-51.

52. Markman M, Cleary S, Pfeifle C et al. Cisplatin administered by the intracavity route for malignant mesotheliomas of the pleura. Cancer 1986; 58: 18-21.

53. Yap BS, Benjamin RS, Burgess MA et al. The value of adriamycin in the treatment of diffuse malignant pleural mesothelioma. Cancer 1978; 42: 1.692-6.

54. Rogoff EE, Hiliaris BS, Havos AG. Long-term survival in patients with malignant peritoneal mesothelioma treated with irradiation. Cancer 1973; 32: 656-64. 